\title{
Asma en la infancia: controversias que conllevan al subdiagnóstico
}

\author{
Asthma in childhood: controversies that lead to \\ underdiagnosis
}

\author{
José Miguel Escamilla ArRieta, MD.(1); María ISAbel Escamilla, MD. ${ }^{(2)}$; \\ CÉsAR ANDRÉs MuÑoz MEJÍA, MD., MSc. ${ }^{(3)}$
}

\section{Resumen}

El asma es la enfermedad crónica más frecuente en la niñez y conduce a múltiples complicaciones, fenómeno que en la actualidad representa un problema de salud pública. El reporte de síntomas bronco-obstructivos es mucho mayor que el diagnóstico médico de asma durante la infancia, hecho que sugiere que hay una tendencia a no reconocer que los niños en esta edad sufren de esta enfermedad y por tanto eventualmente se ocasiona retraso en su tratamiento. El rechazo a clasificar a los pacientes como asmáticos, la falta de un consenso diagnóstico, los múltiples fenotipos, la flexibilidad de las escalas de severidad y el desconocimiento del asma en la infancia como una enfermedad intermitente pero potencialmente grave, son factores del manejo médico que podrían favorecer una alta morbilidad. Esta revisión plantea la necesidad de ser más contundentes en el diagnóstico del asma en la infancia, sin caer en retrasos que permitan iniciar un tratamiento oportuno y adecuado.

Palabras clave: asma, infancia, diagnóstico, tratamiento, fenotipos.

\begin{abstract}
Asthma is the most common chronic disease occurring in childhood. It leads to many complications, and has therefore become a public health concern. Broncho-obstructive symptoms are much more often reported than the medical diagnosis of childhood asthma, which suggests that there is a tendency to disavow the fact that children suffer from this disease. Eventually, this leads to delayed diagnosis. The reluctance to classify patients as suffering from asthma, the lack of diagnostic consensus, the multiple phenotypes, the flexibility of severity scores, and the lack of awareness of childhood asthma as an intermittent but potentially serious disease are factors of medical management that could favor high morbidity. This review states that it is necessary to be more assertive in diagnosing childhood asthma, in order to avoid delayed initiation of adequate treatment.
\end{abstract}

Keywords: asthma, childhood, diagnosis, treatment, phenotypes.
(1)Médico Neumólogo Pediatra. Profesor
titular Departamento de Pediatría, Uni-
versidad de Cartagena. Profesor Posgra-
do de Pediatría, Universidad del Sinú.
Director Departamento de Neumología
y Alergia Pediátrica, Hospital Infantil
Napoleón Franco Pareja. Cartagena, Co-
lombia.
${ }^{(2)}$ Médico Residente de Pediatría. Uni-
versidad Militar Nueva Granada, Hos-
pital Militar Central. Bogotá, Colombia.
(3)Médico Inmunólogo, Residente de Pe-
diatría. Universidad del Sinú, Cartagena
Colombia.
Correspondencia: José Miguel Escami-
lla Arrieta, correo electrónico: josescar@
gmail.com
Recibido: $01 / 06 / 15$. Aceptado: 15/06/15. 


\section{Introducción}

Pese a que el asma se considera la principal enfermedad crónica en la niñez (1), los datos sobre su diagnóstico y las estrategias terapéuticas son muy limitados, adicional a que son pocos los documentos que se han centrado en forma exclusiva a este diagnóstico en la infancia temprana (período de lactancia y pre-escolar), lo cual contribuye a que no se logre un consenso universal respecto a cómo diagnosticar, cuándo y con qué tratar.

Opiniones muy destacadas hablan de la necesidad de un diagnóstico temprano; los clínicos que atienden lactantes con síntomas recurrentes de asma no deberían esperar a que se aclare la patogenia altamente compleja de esta condición para establecer su diagnóstico y tratamiento antiasmático (2), pues el asma crónica iniciada en la niñez se asocia con daño significativo e irreversible de la función pulmonar debido a la inflamación de la vía aérea que ocurre en el momento del crecimiento pulmonar rápido (3).

Si bien la mayoría de los estudios epidemiológicos, incluyendo los llevados a cabo en Colombia, reconocen que el asma del adulto regularmente inicia desde la infancia $(4,5)$ y que el asma en niños es más frecuente y grave que a edades mayores, la misma es poco diagnosticada y consecuentemente poco tratada en este grupo etario $(6,7)$.

\section{Los eufemismos del asma en la infancia}

Un temor, por cierto infundado, a la palabra "asma" parece ser responsable de una paradójica y problemática situación en la salud de la infancia con esta enfermedad en donde se aprecia, tal como lo destaca el Programa Nacional de Educación y Prevención de Asma (National Asthma Education and Prevention Program, NAEPP) (5) cómo en el mundo se usan denominaciones con criterios de eufemismos, como: bronquitis crónica, bronquitis sibilante, hiperreactividad bronquial, neumonía recurrente, infección respiratoria recurrente y reflujo gastro-esofágico para no utilizar el término "asma".

Pareciera que documentos de muy alto valor científico apoyan esta tendencia histórica. La
Sociedad Respiratoria Europea (European Respiratory Society, ERS) (8) reconoce la limitación de las pruebas diagnósticas a estas edades y propone usar el término "sibilancias" para no usar el de asma en niños de esta edad, recomendando el apéndice de "sibilancias episódicas y/o multidisparador", desconociendo la posible progresión y/o variabilidad de la misma a través del tiempo, hacia grados de mayor o menor frecuencia y severidad, y que dependen en mucho de las condiciones ambientales del individuo (9).

La que hoy ha sido referida como la mejor y más consensuada definición de asma en niños, fue descrita hace ya más de dos décadas: La presencia de tos y/o sibilancias en el contexto donde otros diagnósticos menos frecuentes han sido excluidos razonablemente (10-14); esta se ha convertido en la definición más aceptada por los clínicos, porque tiene el gran mérito de no confundir situaciones clínicas con hallazgos anatomo-patológicos, epidemiológicos o fisiológicos que solo son evaluables con pruebas de laboratorio y que no son capaces por sí solas de confirmar o excluir el diagnóstico de asma (15).

La guía NAEPP2 describe textualmente: aunque los episodios recurrentes de tos y sibilancias con mayor frecuencia se deben a la presencia de asma, otras causas, significativamente menos comunes, deben considerarse en la evaluación inicial al igual que cuando no existe una clara respuesta al tratamiento (5). Esta aseveración parece ser, una juiciosa pero también clara aceptación a la definición de asma pediátrica que se mencionó anteriormente y una conceptualización a aquel tradicional adagio que reza "si bien, no todo lo que sibila es asma, eso casi siempre lo es", ya que otras causas son significativamente menos comunes.

Recientemente, la Sociedad Torácica Canadiense (16) emitió un artículo especial donde propone operativamente cuándo y cómo hacer el diagnóstico de asma en preescolares. En esta guía se definen tres criterios fundamentales para el diagnóstico: la documentación objetiva de la bronco-obstrucción, la reversibilidad de esta y el descarte razonable de otros diagnósticos. En estos se prefiere la evidencia 
médica de la bronco-obstrucción y como alternativa el reporte de los cuidadores. Esta evaluación es aplicable para niños con dos o más crisis broncoobstructivas o síntomas sugestivos de asma. Un aspecto interesante de esta publicación es que pese a que las características de atopia del niño y los antecedentes familiares de asma aumentan la sospecha clínica, no son necesarios para el diagnóstico, lo que permite incluir a los niños cuyos síntomas son inducidos por infecciones virales (16).

\section{Identificar al niño con asma en la infancia es más importante que predecirla}

El asma es la enfermedad crónica más prevalente en niños (1) y se encuentra en aumento en todo el mundo (17), especialmente en países en vía de desarrollo como Colombia $(18,19)$. La prevalencia de síntomas de asma en nuestro país se incrementó en un $2 \%$ en 11 años (de 1998 a 2009) $(19,20)$. En ambos períodos se encontró un subdiagnóstico médico en comparación con el reporte paterno de síntomas respiratorios, siendo dicha brecha mayor en los niños más pequeños. Este fenómeno también se ha visto en países desarrollados como Estados Unidos (21). Adicionalmente, el asma es la principal causa de ausentismo escolar (22) y una de las enfermedades que más gastos directos e indirectos acarrean al sistema de salud y a los gobiernos (23).

La exposición temprana y sostenida a un ambiente agresivo (fuertes agentes inhalados) parece determinar un fenotipo de sibilancias y/o asma relacionado comúnmente con infecciones respiratorias virales, que comienza en los primeros tres meses de vida, progresa con episodios más severos y frecuentes de sibilancias y se asocia generalmente con complicaciones como la neumonía (24).

La neumonía es la principal causa de mortalidad en niños menores de cinco años (25) y un alto porcentaje de estos individuos tienen antecedentes de "sibilancias recurrentes" (26); es decir, hay una condición inflamatoria en los niños asmáticos y la neumonía constituye realmente una complicación en ellos. Lo anterior, sumado al subdiagnóstico del asma en la infancia, influyen en la alta morbilidad y mortalidad de la neumonía pero también ofrece un punto de intervención temprana para la prevención de esta enfermedad: si se mejora el control del asma (enfermedad de base) se disminuiría entonces la incidencia de sus complicaciones (procesos infecciosos graves).

A principios de la década de los noventa se publicaron los primeros datos de la cohorte de Tucson, uno de los más importantes hitos en el conocimiento del asma infantil, el cual ha permitido descubrir hechos cruciales relacionados con el desarrollo del asma, sus factores de riesgo así como la caracterización de algunos patrones evolutivos (27). Sin embargo, los tres perfiles de niños afectados de sibilancias: sibilantes precoces transitorios, sibilantes no atópicos y sibilantes asociados a $\operatorname{IgE}$ elevada (o "asmáticos") se han identificado como "fenotipos de asma infantil". Al tratarse de un rasgo que no puede observarse al atender al paciente en la consulta, no puede preverse su evolución hasta cuando cumpla 10 o 12 años.

Los trabajos que estudian la evolución del asma desde el nacimiento documentan que hasta el 50\% de los niños sibilan en la edad preescolar y que aproximadamente el $80 \%$ de ellos deja de tener sibilancias al llegar a la adolescencia, lo que explicaría que algunos autores vean poco lógico considerar que todos tengan que tener asma con inflamación eosinofílica y que deban ser tratados con esteroides inhalados.

El planteamiento que debemos hacernos es el siguiente: si el niño menor de 5 a 6 años tiene historia de sibilancias recurrentes, ¿tiene "asma" ahora? Si se ha hecho un ejercicio clínico para descartar otras enfermedades que cursan con bronco-obstrucción, lo respuesta más probable es SI y debe ser intervenido en ese momento para disminuir todas las complicaciones mencionadas.

Es poco útil denominar solo como asmáticos a los niños que sufren de sibilancias y son atópicos, dejando al resto con un manejo expectante, pues ellos representan una gran carga de morbilidad respiratoria. Lo anterior representa un problema de salud pública en el que el diagnóstico oportuno, las intervenciones adecuadas y las actividades 
de promoción y prevención impactarían de manera positiva en el bienestar de la población infantil. Todas estas son oportunidades de investigación en la actualidad.

\section{La utilidad de clasificar los fenotipos en el asma infantil}

Hoy se reconoce ampliamente que muchos consideran el asma como una enfermedad inflamatoria heterogénea, multigénica y multifactorial, por lo que es apenas obvio que existan diferentes fenotipos de expresión clínica y fisiopatológica, los cuales son el resultado de múltiples factores como la genética, la exposición ambiental, el género, las infecciones, la nutrición, la obesidad, la edad y la epigenética (28-31).

Es así que el término asma debe ser interpretado a la luz que existen muchas formas o fenotipos de asma en el niño pequeño, que no son iguales al asma del adulto, debiendo ser considerado como un síndrome y no como una única enfermedad (29).

Algunos consensos y documentos pediátricos recientes $(13,27-28)$ suponen prioritario un análisis crítico de los fenotipos, aunque también hoy se sabe que las diferentes condiciones fenotípicas no son mutuamente excluyentes, pudiendo coexistir en un mismo individuo. La mayoría de los niños, tanto atópicos como no atópicos, hacen infecciones virales recurrentes y frecuentes, que pueden inducir sibilancias y clínica característica de lo que se ha consensado como asma, lo que inclinaría a establecer el diagnóstico e iniciar el tratamiento oportuno.

Si bien ha sido tradicional explicar la fisiopatología del asma bajo el modelo Th2 (linfocitos $\mathrm{T}$ ayudadores tipo 2) mediada por IgE y eosinófilos, también ha sido documentado el modelo del asma inducida por virus (32) y se evidencia que el asma no atópica (33), en mucho inducida por estas infecciones, alcanza más altas prevalencias y mayor morbilidad en comunidades pobres como la nuestra $(34,35)$.

La evidencia científica (36) sugiere que la inmunidad congénita del individuo podría ser alterada por exposiciones ambientales y biológicas durante los tres primeros meses, lo que daría por resultado un número mayor de episodios de sibilancias durante el primer año, y relaciona exposiciones ambientales más altas con episodios más frecuentes y posiblemente más severos de sibilancias (37). Es probable que los factores de riesgo ambiental como aquellos relacionados con condiciones socioeconómicas bajas, desempeñen un papel en la frecuencia y severidad de las sibilancias y los broncoespasmos de esta población (24).

Un interesante estudio experimental evidencia la importancia del broncoespasmo recurrente, documentando que el inducido por metacolina es igual de lesivo en condicionar remodelación de la vía aérea al de la inflamación eosinofílica persistente inducida por la inhalación de alergenos de ácaros (38).

Un diagnóstico e intervención precoz de la enfermedad resulta clave para hacer tratamientos más eficaces con el fin de preservar el crecimiento y/o función pulmonar y disminuir su alta morbimortalidad. Al aceptar la existencia de diversos fenotipos de asma, se amplía el concepto de la enfermedad como síndrome y abre el debate a diversas posibilidades terapéuticas.

\section{La clasificación del asma infantil: definiendo quién necesita tratamiento farmacológico}

Al establecer el diagnóstico de asma, todas las guías recomiendan iniciar con una clasificación en severidad que permitirá indicar terapias escalonadas en el primer momento.

Las características fisiopatológicas de los lactantes y su estado inmunológico en maduración, con una mayor frecuencia de infecciones virales, permite explicarnos la alta frecuencia y severidad, aunque regularmente intermitentes, de las exacerbaciones asmáticas (39). Por ello nos unimos al concepto de no parecernos apropiado, ni evidenciado, considerar la clasificación en severidad de "persistente leve" en el niño menor con asma (40).

Algunas guías pediátricas que consideran que el asma infantil es una entidad frecuentemente 
intermitente por su condición de ser inducida por infecciones virales, las cuales pueden ser graves, $\mathrm{y}$ la pobre posibilidad de la evaluación de la función pulmonar, así mismo, proponen clasificar la severidad de la enfermedad de acuerdo con los síntomas solamente $(41,42)$ en episódica ocasional, episódica frecuente, persistente moderada y persistente grave, recomendando el uso de tratamientos controladores con base en corticoides inhalados y/o anti-leucotrienos, a partir de los episodios frecuentes.

Es destacable también que en el mismo sentido pudiéramos seguir las pautas descritas por el informe del Panel de Expertos (Expert Panel Report-3) del NAEPP (8) y adaptadas por la Guía de la Asociación Colombiana de Neumología Pediátrica (ACNP) y el Ministerio de Salud de Colombia (14), que recomienda en niños menores de 4 años iniciar el tratamiento de control cuando ocurra cualquiera de las siguientes condiciones:

- Tratamiento sintomático con broncodilatadores más de 2 veces/semana durante un mes.

- Al menos 2 exacerbaciones de asma que requirieron corticoides sistémicos en los últimos 6 meses (intermitentes, pero frecuentes y severas).

- Al menos 4 episodios de sibilancias en el último año y el Índice Predictor de Asma (IPA) es positivo (intermitentes, pero con factor de riesgo de asma persistente alérgica).

En la práctica, la introducción del tratamiento controlador se hará en función de la frecuencia, intensidad de los síntomas y sospecha del fenotipo de asma del niño, pero con el objetivo final del buen control de la enfermedad.

Si bien las guías más reconocidas proponen establecer una clasificación de control de asma basada en opinión de expertos y no en evidencias de historia natural del asma o validación de las mismas, un documento denominado TRACK proporciona la primera herramienta validada para evaluar el asma en niños menores de 4 años de edad (43). Este instrumento con un cuestionario sencillo y práctico, de cinco preguntas, que se hacen a los padres o cuidadores del menor, categoriza solo dos niveles de control: controlado y/o no controlado. $\mathrm{Si}$ el resultado es 80 o menos (de un total de 100), el asma no está bajo control y motivará al médico y a los cuidadores a revisar la terapéutica instaurada para ascender en la terapia y procurar lograr el control de la misma.

\section{Las ventajas del tratamiento temprano}

Pese a que los estudios son escasos, un trabajo realizado hace más de dos décadas (44), documentó una relación significativa inversa entre la duración del asma antes del tratamiento con budesonida y el incremento anual del $\mathrm{VEF}_{1}$ después de 3 años de tratamiento con esta. Los enfermos que empezaron el tratamiento después de 5 años del comienzo de la enfermedad obtuvieron un $\mathrm{VEF}_{1}$ significativamente menor que el de los niños que lo empezaron en los dos primeros años del comienzo de la misma.

Este estudio observó al tiempo una reducción significativa del número de ingresos hospitalarios por agudización grave del asma en los niños que recibían esteroides a edades más tempranas. Con base en lo anterior, el tratamiento temprano con anti-inflamatorios podría prevenir la declinación de la función pulmonar que ocurre a través del tiempo en los pacientes asmáticos y su gran morbilidad. El mensaje de este trabajo es que el tratamiento debe ser iniciado de manera precoz, y probablemente esta intervención ofrezca más beneficios que riesgos (44).

Otro estudio donde participaron 285 niños asmáticos persistentes con IPA positivo y con un tiempo mayor a dos años de enfermedad activa, mostró que el tratamiento diario con fluticasona inhalada se asoció con una mejoría clínica significativamente mayor en comparación con el grupo placebo, y al retirar los esteroides a los dos años de tratamiento el grupo con corticoides inhalados (CI) tuvo un índice de recaídas similar a los que no lo recibieron. Lo anterior demuestra la eficacia de los CI en niños con asma persistente atópicos (45).

Los fármacos disponibles que mejor han demostrado y categorizado su utilidad en el control del asma en menores de 4 años son los CI y los 
antagonistas de los leucotrienos. Al evaluar distintas revisiones, algunas sugieren más a los CI cuando el fenotipo inflamatorio es eosinofílico, el IPA es positivo y la severidad es más persistente y severa $(46,47)$, mientras que los antagonistas de los receptores de los leucotrienos tendrían mejor respuesta cuando el fenotipo inflamatorio es de predominio neutrofílico o inducido por virus, lo que encaja mejor en las formas episódicas, en niños menores y con corto tiempo de evolución (48).

Sin embargo, un metaanálisis de 29 ensayos clínicos aleatorizados, que estudió la eficacia de los CI en 3.592 niños menores de 5 años, con sibilancias recurrentes o asma, concluyó que reducen las exacerbaciones cerca de un $40 \%$, al compararlos con placebo, independientemente de parámetros la edad, diagnóstico (sibilancias o asma), presencia de atopia, modo de dispensación del esteroide y CI utilizado. Los autores proponen que se podría realizar un ensayo terapéutico con CI en estos pacientes, independientemente del fenotipo, a pesar de ser menos probable la eficacia en los niños con "sibilancias" que en los "asmáticos" atópicos (49).

Un metaanálisis reciente evidenció que la terapia con $\mathrm{CI}$ inhalados debe preferirse sobre otros medicamentos (antagonistas de los leucotrienos, $\beta$-adrenérgicos de acción prolongada y corticoides orales) y que su utilización diaria es mejor que la intermitente para disminuir los síntomas respiratorios cotidianos $(50)$.

Actualmente muchas preguntas siguen sin respuesta, las cuales incluyen el tratamiento adecuado para cada paciente (eficacia/efectividad/ seguridad), tipo de horario (regular versus según sea necesario) y la dosis (dosis alta versus dosis baja).

\section{Conclusiones}

El asma durante la infancia, en lactantes y preescolares, es una enfermedad frecuentemente subdiagnosticada. Algunos factores de la práctica médica como la utilización de eufemismos para el asma, no reconocer los potenciales riesgos y complicaciones que tiene esta población y la falta de homogeneidad en los criterios diagnósticos, en especial en nuestro medio donde se ha evidenciado mayor severidad, gastos y complicaciones fatales como la neumonía, predisponen a que consecuentemente haya un inicio tardío del tratamiento.

Es necesario establecer criterios diagnósticos que incluyan a todos los fenotipos de asma durante estas edades para poder reconocer oportunamente al niño que necesita ser intervenido con medicamentos.

\section{Conflictos de interés}

Los autores declaran no tener conflictos de interés.

\section{Bibliografía}

1. Centers for Disease Control and Prevention, National Health Interview Survey--2011. Available online Last accessed. $2015 ; 1: 143$.

2. Mallol J. Asma del lactante: Bases para el diagnóstico y tratamiento. Pulmão RJ. 2013;22(3):48-52.

3. Martinez FD. Present and future treatment of asthma in infants and young children. J Allerg Clin Immunol. 1999;104(4 Pt 2):169-74.

4. Martinez FD. The origins of asthma and chronic obstructive pulmonary disease in early life. Proc Am Thorac Soc. 2009;6(3):272-7.

5. National Asthma Education Prevention Program. Expert Panel Report 3 (EPR-3): Guidelines for the Diagnosis and Management of Asthma-Summary Report 2007. J Allerg Clin Immunol. 2007;120(5 Suppl):S94-138.

6. Caraballo L, Cadavid A, Mendoza J. Prevalence of asthma in a tropical city of Colombia. Ann Allerg. 1992;68(6):525-9.

7. Dennis RJ, Caraballo L, Garcia E, Rojas MX, Rondon MA, Perez A, et al. Prevalence of asthma and other allergic conditions in Colombia 2009-2010: a cross-sectional study. BMC Pulmonary Medicine. 2012;12:17.

8. Brand PL, Baraldi E, Bisgaard H, Boner AL, Castro-Rodriguez JA, Custovic A, et al. Definition, assessment and treatment of wheezing disorders in preschool children: an evidence-based approach. Eur Resp J. 2008;32(4):1096-110.

9. Schultz A, Brand PL. Episodic viral wheeze and multiple trigger wheeze in preschool children: a useful distinction for clinicians? Paediat Resp Rev. 2011;12(3):160-4.

10. Phelan PD OA, Oswald H. Asthma: classification, clinical patterns and natural history. Clinical paediatrics. London: Ed. Bailliere Tindall; 1995.

11. Warner JO, Naspitz CK. Third International Pediatric Consensus statement on the management of childhood asthma. International Pediatric Asthma Consensus Group. Pediat Pulmonol. 1998;25(1):1-17. 
12. V. M. Evidence-based paediatrics and child health. London, UJ: BMJ Books; 2000.

13. Castillo Laita JA, De Benito Fernández J, Escribano Montaner A, Fernández Benítez M, García de la Rubia S, Garde Garde J, et al. Consenso sobre tratamiento del asma en pediatría. Anal Pediat. 2007;67(3):253-73.

14. ACNP. Guía de práctica clínica (GPC) para el diagnóstico, atención integral y seguimiento de niños y niñas con diagnóstico de asma. 2013.

15. Vega-Briceño LE, Prado F, Sánchez I. Fenotipos de asma en el lactante y preescolar. Boletín de la Escuela de Medicina. 2005;30(2):26-30.

16. Ducharme FM, Dell SD, Radhakrishnan D, Grad RM, Watson WT, Yang CL, et al. Diagnosis and management of asthma in preschoolers: A Canadian Thoracic Society and Canadian Paediatric Society position paper. Can Resp J. 2015;22(3):135-43.

17. Masoli M, Fabian D, Holt S, Beasley R. The global burden of asthma: executive summary of the GINA Dissemination Committee report. Allergy. 2004;59(5):469-78.

18. Pearce N, Aït-Khaled N, Beasley R, Mallol J, Keil U, Mitchell $\mathrm{E}$, et al. Worldwide trends in the prevalence of asthma symptoms: phase III of the International Study of Asthma and Allergies in Childhood (ISAAC). Thorax. 2007;62(9):75866.

19. Rodriguez C, Sossa M, Goss CH. Factors associated with severe disease in a population of asthmatic children in Bogotá, Colombia. J Asthma. 2008;45:141-147.

20. Dennis R, Caraballo L, García E, Caballero A, Aristizábal G, Córdoba $\mathrm{H}$, et al. Asthma and other allergic conditions in Colombia: a study in 6 cities. Ann Allerg, Asthma \& Immunol. 2004;93(6):568-74.

21. Clark BR, Burkett SA, Andridge RR, Buckley TJ. Evidence of high rates of undiagnosed asthma in central Ohio elementary schoolchildren. J School Health. 2013;83(12):896-906.

22. Akinbami LJ, LaFleur BJ, Schoendorf KC. Racial and income disparities in childhood asthma in the United States. Ambulatory Pediatrics. 2002;2(5):382-7.

23. Murthy K, Sastry J. Economic burden of asthma. Burden of Disease in India. 2005:251.

24. Mallol J, Andrade R, Auger F, Rodriguez J, Alvarado R, Figueroa L. Wheezing during the first year of life in infants from low-income population: a descriptive study. Allergologia et Immunopathologia. 2005;33(5):257-63.

25. Bryce J, Boschi-Pinto C, Shibuya K, Black RE, Group WCHER. WHO estimates of the causes of death in children. The Lancet. 2005;365(9465):1147-52.

26. Cabezuelo HG, Vidal MS, Abeledo GA, Frontera IP, editors. [Underlying causes of recurrent pneumonia]. Anal Pediat. (Barcelona, Spain: 2003); 2005.

27. Martinez FD, Wright AL, Taussig LM, Holberg CJ, Halonen
M, Morgan WJ. Asthma and wheezing in the first six years of life. The Group Health Medical Associates. New Eng J Med. 1995;332(3):133-8.

28. Bacharier LB, Boner A, Carlsen KH, Eigenmann PA, Frischer T, Gotz M, et al. Diagnosis and treatment of asthma in childhood: a PRACTALL consensus report. Allergy. 2008;63(1):5-34.

29. Castro-RoDRíguez J. Factores de riesgo para asma infantil. Neumol Pediat. 2006;1:55-8.

30. Bush A. Phenotype specific treatment of asthma in childhood. Paediat Resp Rev. 2004;5 Suppl A:S93-101.

31. Townshend J, Hails S, McKean M. Management of asthma in children. BMJ. 2007;335(7613):253-7.

32. Gern JE. Viral respiratory infection and the link to asthma. Pediat Infect Dis J. 2008;27(10 Suppl):S97-103.

33. Just J, Fournier L, Momas I, Zambetti C, Sahraoui F, Grimfeld A. Clinical significance of bronchoalveolar eosinophils in childhood asthma. J Allerg Clin Immunol. 2002;110(1):424.

34. Weinmayr G, Weiland SK, Bjorksten B, Brunekreef B, Buchele G, Cookson WO, et al. Atopic sensitization and the international variation of asthma symptom prevalence in children. Am J Resp Crit Care Med. 2007;176(6):565-74.

35. Penny ME, Murad S, Madrid SS, Herrera TS, Pineiro A, Caceres DE, et al. Respiratory symptoms, asthma, exercise test spirometry, and atopy in schoolchildren from a Lima shanty town. Thorax. 2001;56(8):607-12.

36. Garcia-Marcos L. EISL study (Estudio Internacional de Sibilancias en Lactantes): A global perspective of wheezing during the first year of life. Allergologia et immunopathologia. 2016;44(1):1-2.

37. Guerra S, Lohman IC, Halonen M, Martinez FD, Wright AL. Reduced interferon gamma production and soluble CD14 levels in early life predict recurrent wheezing by 1 year of age. Am J Resp Crit Care Med. 2004;169(1):70-6.

38. Grainge CL, Lau LC, Ward JA, Dulay V, Lahiff G, Wilson S, et al. Effect of bronchoconstriction on airway remodeling in asthma. New Eng J Med. 2011;364(21):2006-15.

39. Warner JO, Gotz M, Landau LI, Levison H, Milner AD, Pedersen S, et al. Management of asthma: a consensus statement. Arch Dis Childhood. 1989;64(7):1065-79.

40. Bacharier LB, Phillips BR, Bloomberg GR, Zeiger RS, Paul IM, Krawiec M, et al. Severe intermittent wheezing in preschool children: a distinct phenotype. J Allergy Clin Immunol. 2007;119(3):604-10.

41. Moral VP. GEMA 4.0. Guía española para el manejo del asma. Arch Bronconeumol. 2015;51:2-54.

42. Guía de práctica clínica en el tratamiento del niño con asma. Guía No 4. Eds.: Álvaro Posada Díaz, William Parra Cardeño. Segunda ed. Bogotá. Asociación Colombiana de Neumología Pediátrica; 2010. p. 17. 
43. Murphy KR, Zeiger RS, Kosinski M, Chipps B, Mellon $\mathrm{M}$, Schatz M, et al. Test for respiratory and asthma control in kids (TRACK): a caregiver-completed questionnaire for preschool-aged children. J Aallerg Clin Immunol. 2009;123(4):833-9 e9.

44. Agertoft L, Pedersen S. Effects of long-term treatment with an inhaled corticosteroid on growth and pulmonary function in asthmatic children. Resp Med. 1994;88(5):373-81.

45. Guilbert TW, Morgan WJ, Zeiger RS, Mauger DT, Boehmer SJ, Szefler SJ, et al. Long-term inhaled corticosteroids in preschool children at high risk for asthma. New Eng J Med. 2006;354(19):1985-97.

46. Castro-Rodriguez JA, Rodrigo GJ. Efficacy of inhaled corticosteroids in wheezy infants/preschoolers. Resp Med. 2013;107(1):153-4.
47. Merkus PJ, de Jongste JC. Inhaled corticosteroids in wheezy infants. Am J Respir Crit Care Med. 2005;172(8):1058-9; author reply 9.

48. Warner J, Naspitz C, Cropp G. Third International Pediatric Consensus statement on the management of childhood asthma. Pediat Pulmonol. 1998;25(1):1-17.

49. Castro-Rodriguez JA, Rodrigo GJ. Efficacy of inhaled corticosteroids in infants and preschoolers with recurrent wheezing and asthma: a systematic review with metaanalysis. Pediatrics. 2009;123(3):e519-25.

50. Castro-Rodriguez JA, Custovic A, Ducharme FM. Treatment of asthma in young children: evidence-based recommendations. Asthma Research and Practice. 2016;2(1):1. 